\title{
A Catalogue of Combinatorial Geometries
}

\author{
By John E. Blackburn, Henry H. Crapo and Denis A. Higgs
}

\begin{abstract}
We include in the microfiche section of this issue a catalogue of all the different geometric configurations which may be constructed from $n$ points, $n \leqq 8$, by specifying their points, lines, planes, . . . colines, copoints, their flats of each rank. It suffices to list the copoints of each combinatorial geometry $G$, because the colines of $G$ are the copoints of a geometry earlier in the list, which may be located by deleting one component of the designator of $\boldsymbol{G}$.
\end{abstract}

1. Introduction. We list, in the microfiche section of this issue, all the different geometrical configurations which may be constructed with $n$ points, $n \leqq 8$.

Combinatorial geometries [2] are very general, including the projective, affine, and Möbius (etc.) geometries over various fields, any subgeometry (determined by a subset of the set of points) of such a geometry, as well as many geometries that are not so representable. The flats of a combinatorial geometry satisfy the following simple axioms: Each flat is a set of points, the empty set $\varnothing$ and the one-point sets are flats, any intersection of flats is a flat, and

the points not in a flat $Y$ (of rank $k$, say)

are partitioned by inclusion in the flats of

rank $k+1$ which contain the flat $Y$.

The theory of single-point extensions of geometric lattices [1] provides a scheme for computer generation of combinatorial geometries. Using that scheme, we verified the catalogue [5] of the 26 geometries on 6 points and of the 101 geometries on 7 points and used it as the input for the next stage in generation. We compared the resulting geometries pairwise for isomorphism, and retained one representative of each isomorphism class. Finally, we made the catalogue consistent with respect to the truncation operation: The colines of any listed geometry are also the copoints of another listed geometry. This enables us to give complete information about each geometric lattice, using a single typed line for each geometry.

The information in this catalogue is relevant to a variety of current research efforts. It is intended primarily as a source of counterexamples. ${ }^{*}$ The theory of

Received February 9, 1970.

AMS (MOS) subject classifications (1970). Primary 05B35.

Key words and phrases. Combinatorial geometries, geometric lattice, configuration, catalogue of geometries, modular filter, Tutte polynomial.

* Readers desiring a copy of this catalogue on punched cards, for computational use, should communicate directly with the authors. 
combinatorial geometries has fortunately progressed now to a point where the geometries on 5 points, and to some extent the graphically-representable geometries, no longer suffice for checking conjectures.

Some typical unsolved problems which may become more accessible after a close look at these small geometries, are the following:

(a) Provide a general coordinatization scheme for combinatorial geometries, and characterize those coordinatizable over various fields (known for $G F(2)$ and GF(3)).

(b) Characterize the geometries (or subgeometries of the geometries) $A_{k}^{n}$, the lattices of varieties of $n$-dimensional space over a field $F$, varieties determined by polynomials in $n$ variables, degree $\leqq k$.

(c) Characterize the subgeometries of Dilworth completions of lower $k$-truncations of free geometries (known for $k=0$, the free geometries, and for $k=1$, the graphic geometries).

(d) Characterize simplicial geometries.

(e) Investigate the chromatic number, or critical exponent, of a geometry with respect to a field over which it is coordinatizable.

For a discussion of geometries, and for definitions of terms used in this paper, we refer the reader to the monograph Combinatorial Geometries [2].

2. Using the Catalogue. Geometries are listed in increasing order by number of points, then by rank. Geometries of equal cardinality and rank are arranged in an order reflecting increasing triviality (points in more general position).

The listing for each geometry of cardinality $n$ and rank $k$ begins with the designator sequence

$$
\begin{array}{lllllll}
1 & n & f_{2} & f_{3} & \cdots & f_{k-1} & 1,
\end{array}
$$

where $f_{i}$ is the number of flats of rank $i$. The digit $f_{i}$ is followed by a letter if there is more than one possible configuration with the sequence

$$
\begin{array}{llllll}
1 & n & f_{2} & \cdots & f_{i} & 1
\end{array} .
$$

Following the designator, we list the nontrivial copoints of the geometry, each copoint being given as a set of points. The trivial copoints in a geometry of rank $k$ are those consisting of exactly $k-1$ points, the least possible number. The concluding entry " $m\left(\begin{array}{c}n \\ k-1\end{array}\right)$ " in each copoint list indicates for each geometry of rank $k$ on $n$ points, the number of trivial copoints. These can easily be reconstructed by hand, because every $k-1$ element subset of the point set must be in at least one copoint. The listing of copoints provides immediate access to the point-copoint incidence relation which determines the geometry. (Recall that the set-theoretic complements of the copoints are the bonds of the geometry, i.e., the circuits of the orthogonal geometry.)

In order to draw the Hasse diagram of the geometric lattice of a geometry with designator

$$
1822 c \quad 19 b \quad 1 \text {, }
$$

say, we start with the unit element " $A B C D E F G H$ " at the top, and list all the copoints 
(including trivial copoints) on a line beneath this. The colines (in this case, the lines) are given earlier in the catalogue with designator

$$
1822 c 1 .
$$

The coplanes occur still earlier, with designator

$$
181 \text {, }
$$

etc. If each flat is joined to those flats of the next higher rank which contain it, the Hasse diagram is completed.

To draw the associated geometric configuration, begin with complete information about the nontrivial flats of various ranks. The nontrivial lines in the geometry

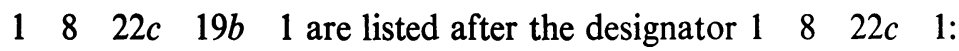

$$
A B C \quad A E D \quad D F G \text {. }
$$

We begin by drawing these in, as in Fig. 1 .

- $H$

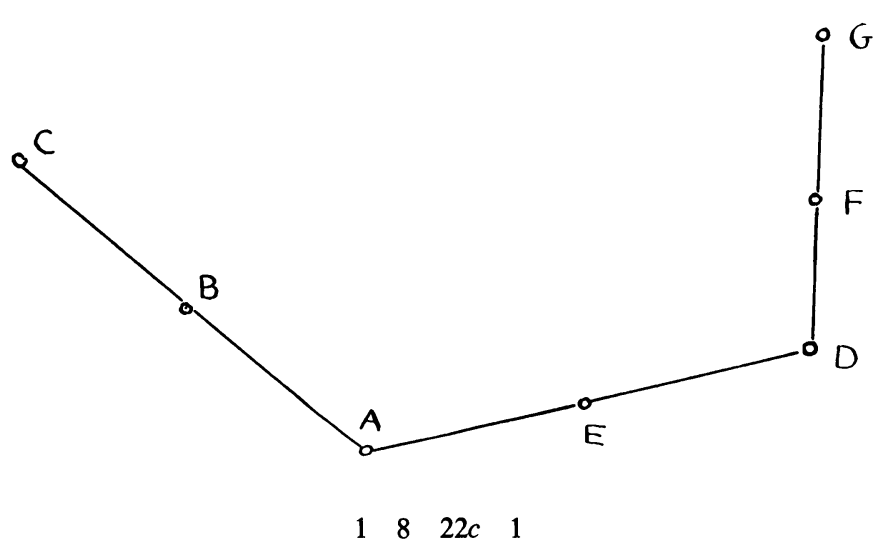

FIGURE 1

The geometry will now be completely determined, once we ascertain whether the lines $A B C$ and $D F G$ are coplanar and determine the position of the point $H$.

Turning now to the list of nontrivial planes, after the designator $1822 c$ $19 b 1$, we see that $A B C$ and $D F G$ are not coplanar. All the planes such as $A B C F$ and $A B C D E$ are predictable. The only new information is given by the planes

$$
B E F H \text { and } C E G H \text {. }
$$

If we redraw our 7 point geometry $A B C D E F G$ in such a way that the planes $B E F$ and $C E G$ intersect in a clearly recognizable line, we may now place the point $H$ anywhere on that line, just so long as it does not line up with any pair of points. 


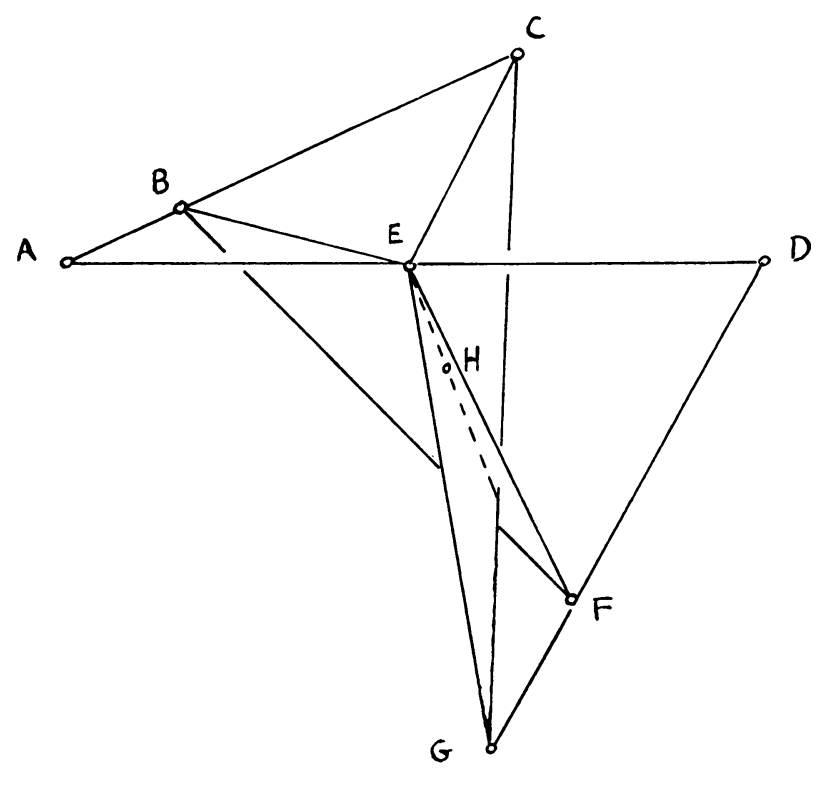

$\begin{array}{lllll}1 & 8 & 22 c & 19 b & 1\end{array}$

FIGURE 2

\section{Observations.}

(a) Representability. Among the 8-point geometries are some that are relevant to questions on representability. By taking a failed Pappus configuration, we obtain a geometry on 9 points which is not representable over any field and, by taking a failed Desargues configuration, we obtain a geometry on 10 points which is not representable over any division ring. Ingleton [6] had conjectured that every geometry on $k$ points is representable over some field if $k \leqq 8$ and over some division ring if $k \leqq 9$. An inspection of the geometries on 7 or fewer points shows that these are all representable over some field-indeed, except for the 7-point projective plane and its orthogonal, they are all representable over the rationals. However, among the 8-point geometries occur some, such as $\begin{array}{llllll}1 & 8 & 28 & 17 & 1 \text {, which are not repre- }\end{array}$ sentable over any division ring. This is a consequence of the fact that every subgeometry $G$ of a projective geometry has the following property: Whenever 4 distinct lines of $G$ are such that no 3 of the lines are coplanar, but the 4 lines are coplanar in pairs with the possible exception of one of the 6 pairs, then the 2 lines of this remaining pair are also coplanar. Since $11 \quad 8 \quad 28 \quad 17 \quad 1$ fails to possess this property, it is not isomorphic to any subgeometry of a projective geometry and is therefore not representable over any division ring. Incidentally, $11 \quad 8 \quad 28 \quad 17 \quad 1$ may be regarded as the affine geometry on 8 points over $\mathrm{GF}(2)\left(\begin{array}{lllll}1 & 8 & 28 & 14 & 1\end{array}\right)$ in which one of the planes has been broken into 4 trivial planes. Richard Rado has described a similar 8-point nonrepresentable geometry, but with more broken planes. Another 8-point geometry which has been mentioned in connection with repre- 
sentation problems is $1 \quad 8 \quad 12 a \quad 1$. This is one of the classical configurations and is known to be representable over the complex numbers but not over the real numbers.

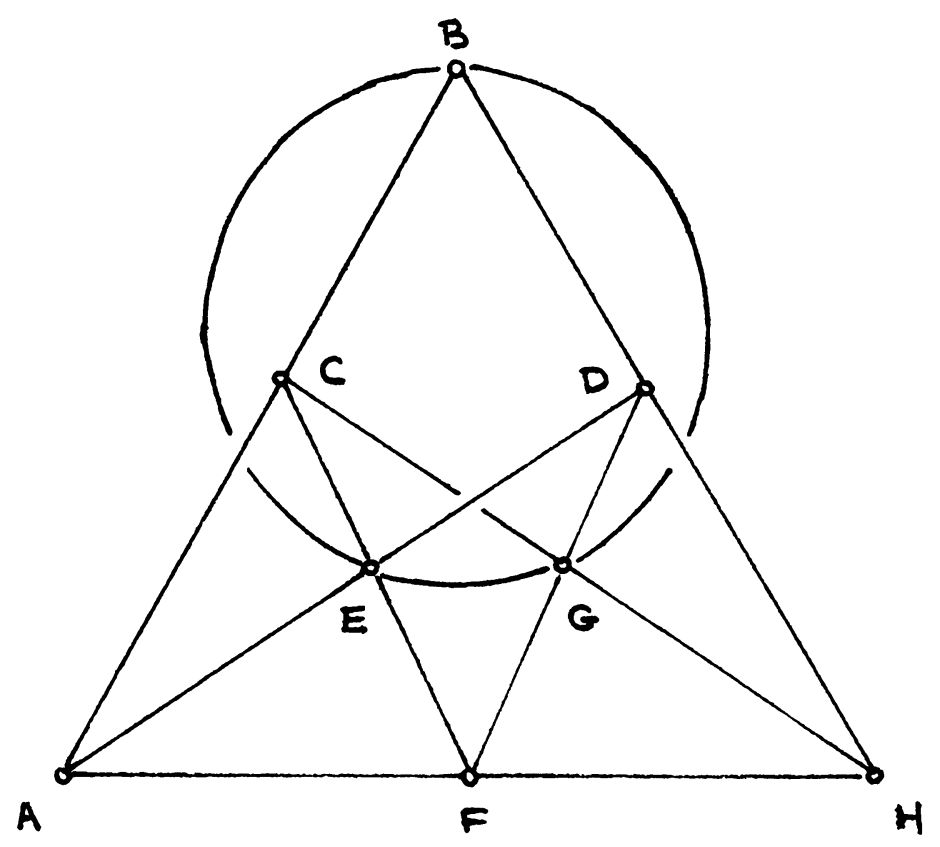

$\begin{array}{llll}1 & 8 & 12 a & 1\end{array}$

A $B$ B E C C G F H

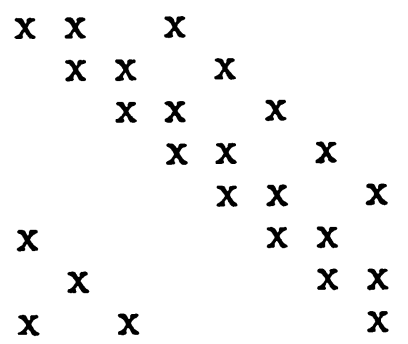

FIGURE 3

(b) Geometries with Equal Polynomials. The catalogue reveals many different geometries having the same Tutte polynomial [3]. The first example is on six points,

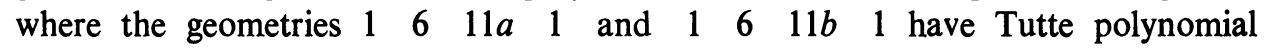




$$
\alpha(x, y)=x^{3}+3 x^{2}+4 x+2 x y+4 y+3 y^{2}+y^{3} .
$$

(Both these geometries are isomorphic to their orthogonal geometries, so the Tutte polynomial has $x-y$ symmetry.)
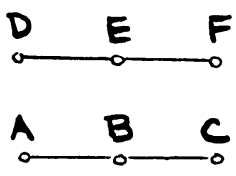

$\begin{array}{llll}1 & 6 & 11 a & 1\end{array}$

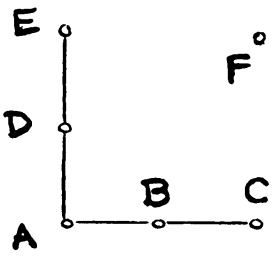

$\begin{array}{llll}1 & 6 & 11 b & 1\end{array}$

Figure 4

Geometries have the same Tutte polynomial if and only if they have the same numbers $s_{i j}$ of subsets with $i$ points and rank $j$, for all $i, j$. Geometries with equal Tutte polynomials have lattices with the same characteristic polynomials, and so do their orthogonal geometries. They also have the same numbers of coboundaries and cycles with kernels of given rank, and images of given size, whenever these constructions are relevant.

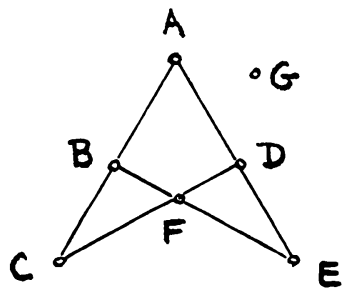

$1713 a \quad 1$

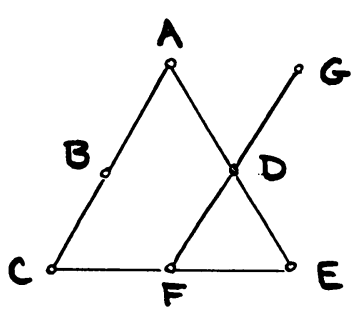

$\begin{array}{llll}1 & 7 & 13 b & 1\end{array}$

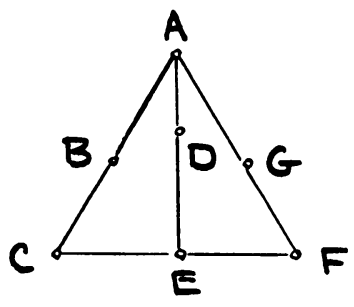

$1713 c \quad 1$

Figure 5

On seven points, the geometries $17 \begin{array}{llllllllll} & 73 a & 1, & 1 & 7 & 13 b & 1 \text {, and } & 1 & 7\end{array}$ 13c 1 all have the Tutte polynomial

$$
x^{3}+4 x^{2}+6 x+4 x y+6 y+6 y^{2}+3 y^{3}+y^{4} .
$$

(c) Isomorphic Extensions from Nonisomorphic Cuts. We generated 8-point geometries by taking the distinct 7-point geometric lattices one at a time, searching for all modular cuts on them, and producing the corresponding single-point extensions, in which the added point depends upon the flats which are in the cut. Consider a situation in which, for two modular cuts $M, N$ on a geometry with lattice $L$, there is an automorphism of the geometry (a permutation of the points of $L$ which carries flats to flats) carrying the cut $M$ onto the cut $N$. In that event, the associated extensions will be isomorphic (under an isomorphism which fixes the added point). It seemed reasonable to suspect that if there were no such automorphism, then the 
extensions of the lattice $L$ by the cuts $M$ and $N$ would be extensions of the same geometry in two essentially different ways, and therefore would be nonisomorphic. But this is not true.

Before we give a counterexample, let us rephrase the above conjecture in such a way as to make it appear much less likely. Given two $(n-1)$-point subgeometries $H=G-e$ and $H^{\prime}=G-e^{\prime}$ in an $n$-point geometry $G$, consider the modular cuts $M$ and $M^{\prime}$ used to extend $H$ and $H^{\prime}$ to $G$. There is an isomorphism from $H$ to $H^{\prime}$, carrying the cut $M$ onto the cut $M^{\prime}$ if and only if there is an automorphism of $G$ which carries $H$ onto $H^{\prime}$ (that is, if and only if $H$ and $H^{\prime}$ are conjugate in $G$ ). Thus, the above situation arises precisely when the subgeometries $H$ and $H^{\prime}$ are conjugate subgeometries of $G$. The (false) conjecture then reads: If two $(n-1)$-point subgeometries of an $n$-point geometry are isomorphic, they are conjugate. Put this way, the conjecture sounds appropriately dubious.

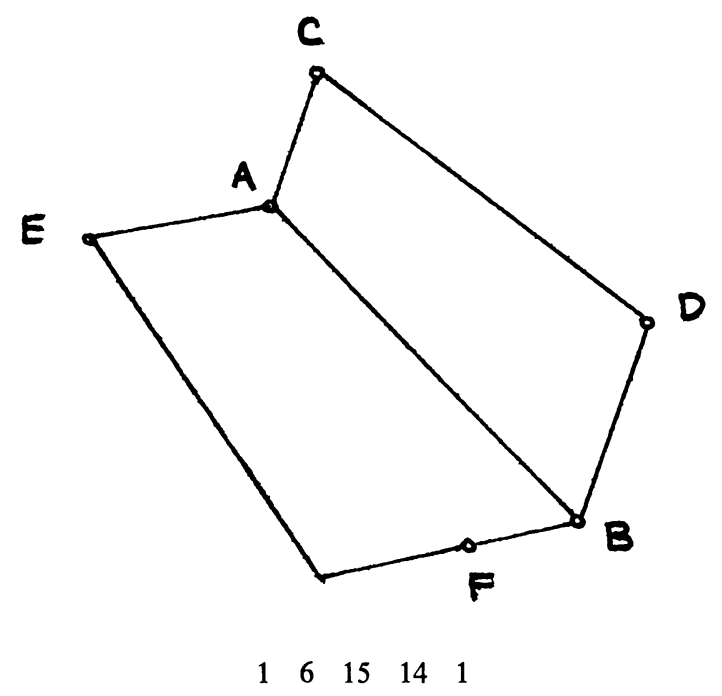

Figure 6

In the geometry $1 \quad 6 \quad 15 \quad 14 \quad 1$, we form modular cuts $M$ and $N$ as follows:

$$
\begin{array}{cc}
M & =\{A C E, \quad B D F, \quad A B C D E F\}, \\
N & =\{C D E, \quad B D F, \quad A B C D E F\} .
\end{array}
$$

No automorphism can carry $M$ into $N$, because the planes in $N$ intersect, those in $M$ do not. The extensions are both isomorphic to the geometry $1 \quad 7 \quad 21 \quad 23 c \quad 1$, as indicated in Fig. 7.

We have a geometry on seven points, containing two 6-point subgeometries which are isomorphic but not conjugate.

(d) The Number of Geometries. Tabulated below are the numbers of nonisomorphic geometries of rank $k$ on $n$ points, for $1 \leqq k \leqq n \leqq 8$. 

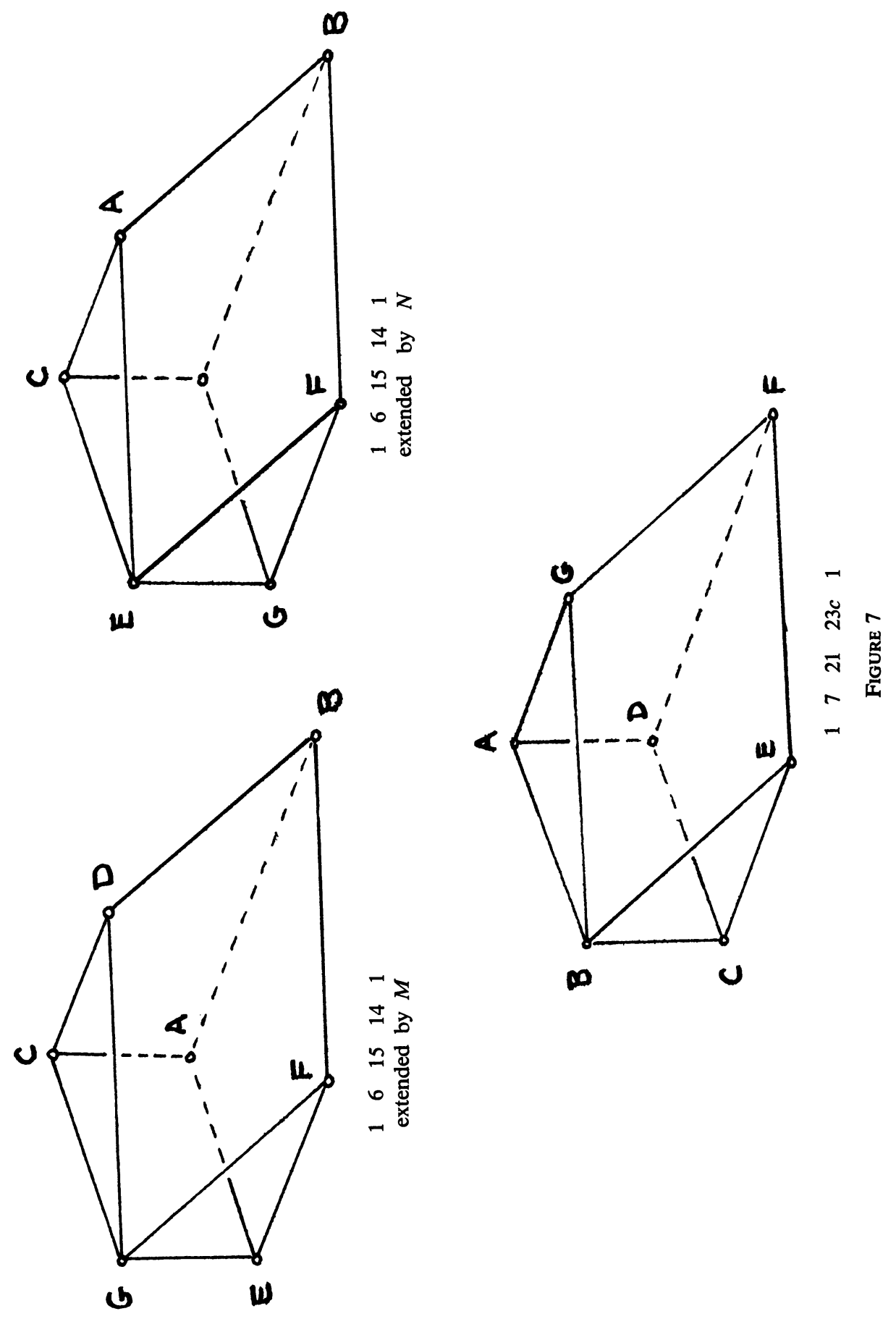


$\begin{array}{cccccccccc} & & 8 & 7 & 6 & 5 & 4 & 3 & 2 & 1 \\ & & \text { pts } & \text { pts } & \text { pts } & \text { pts } & \text { pts } & \text { pts } & \text { pts } & \text { pt } \\ \text { rank } & 1 & . & . & . & . & . & . & . & 1 \\ & 2 & 1 & 1 & 1 & 1 & 1 & 1 & 1 & \\ & 3 & 68 & 23 & 9 & 4 & 2 & 1 & & \\ & 4 & 617 & 49 & 11 & 3 & 1 & & & \\ 5 & 217 & 22 & 4 & 1 & & & & \\ 6 & 40 & 5 & 1 & & & & & \\ 7 & 6 & 1 & & & & & & \\ 8 & 1 & & & & & & & \end{array}$

We note that geometries of rank equal to or just greater than $n / 2$ soon predominate, as $n$ increases. An enumeration of the catalogue entries $1 \quad 8 \quad 28 f_{3} \quad 1$ also reveals that among the 617 geometries of rank $n / 2$ on $n=8$ points, 322 have only trivial colines. These are the geometries associated with Hartmanis partitions of type 3 , in [2].

Doyen [4] has given the number of plane geometries on nine points as 383 . Doyen has proved that these are at least $2^{n}$ nonisomorphic planes on $n$ points, for $n \geqq 10$.

Welsh [9] conjectures that

$$
f_{m+n}>f_{m} \cdot f_{n}
$$

where $f_{n}$ is the number of nonisomorphic pregeometries on $n$ elements. This is surely correct.

This catalogue exhibits the rapid growth of the number $g(n)$ of geometries on $n$ points. Piff and Welsh [8] have now replaced the Crapo-Rota estimate [2]

$$
g(n+1) \simeq(g(n))^{3 / 2}, \quad g(n) \simeq k^{\left((3 / 2)^{n}\right)}
$$

by proven asymptotic lower bounds $b_{\lambda}$, for any $\lambda<1$,

$$
(\exists N, \forall n>N) \quad g(n)>b_{\lambda}(n)=2^{\left(2^{\lambda n}\right)},
$$

which is even closer to the absolute upper bound $2^{(2 n)}$ for structures which are determined by families of subsets of a set. Piff and Welsh give an even larger asymptotic lower bound

$$
(\exists N, \forall n>N) \quad g(n)>n^{\left(2^{\left.n /\left(n^{5 / 2}\right)\right)}\right.} .
$$

It is unlikely that a complete tabulation of 9-point geometries will be either feasible or desirable, as there will be many thousands of them.** The recursion $g(9)=(g(8))^{3 / 2}$ predicts 29260 . We must caution projective plane theorists that this program is not recommended for the construction of a projective plane on 111 points!

4. On the Program. Of the three stages in the production of the catalogue: search for modular cuts, construction of the corresponding lattice extension, and isomorphism check, the first stage requires the most computer time. We tried in a

** The Piff-Welsh lower bound gives no information concerning $g(9)$, because it asserts merely that $g(8) \geqq 19, g(9) \geqq 103$. 
variety of ways to reduce that time demand, and evolved a number of procedures, two of which we now describe.

At one stage in the development of the program, we thought it advisable to search through all subsets of the copoint sets of the 7-point geometries, generating from each the smallest modular cut containing that subset. We could eliminate all subsets which generate modular cuts containing any point, because the associated extensions leave the geometry unchanged (merely doubling a point, or placing a point in the closure of the empty set). Once such a subset is found, it is clearly futile to : inspect any subsets containing it.

This sort of situation occurs frequently in combinatorial problems. We wish to follow a circular path through $2^{n}$ points (representing the subsets of an $n$-element set), but wish to omit those points which represent supersets of sets at which we have already obtained trivial results. Consider the following flow diagram. Subsets of an $n$-element set are indicated as usual by $n$-place binary numbers. Choice " $A$ " is made 0 or 1 in accordance with the last digit in the binary number. Choice " $B$ " is made 1 if the subset just checked gave trivial results (so that all larger subsets should be ignored).

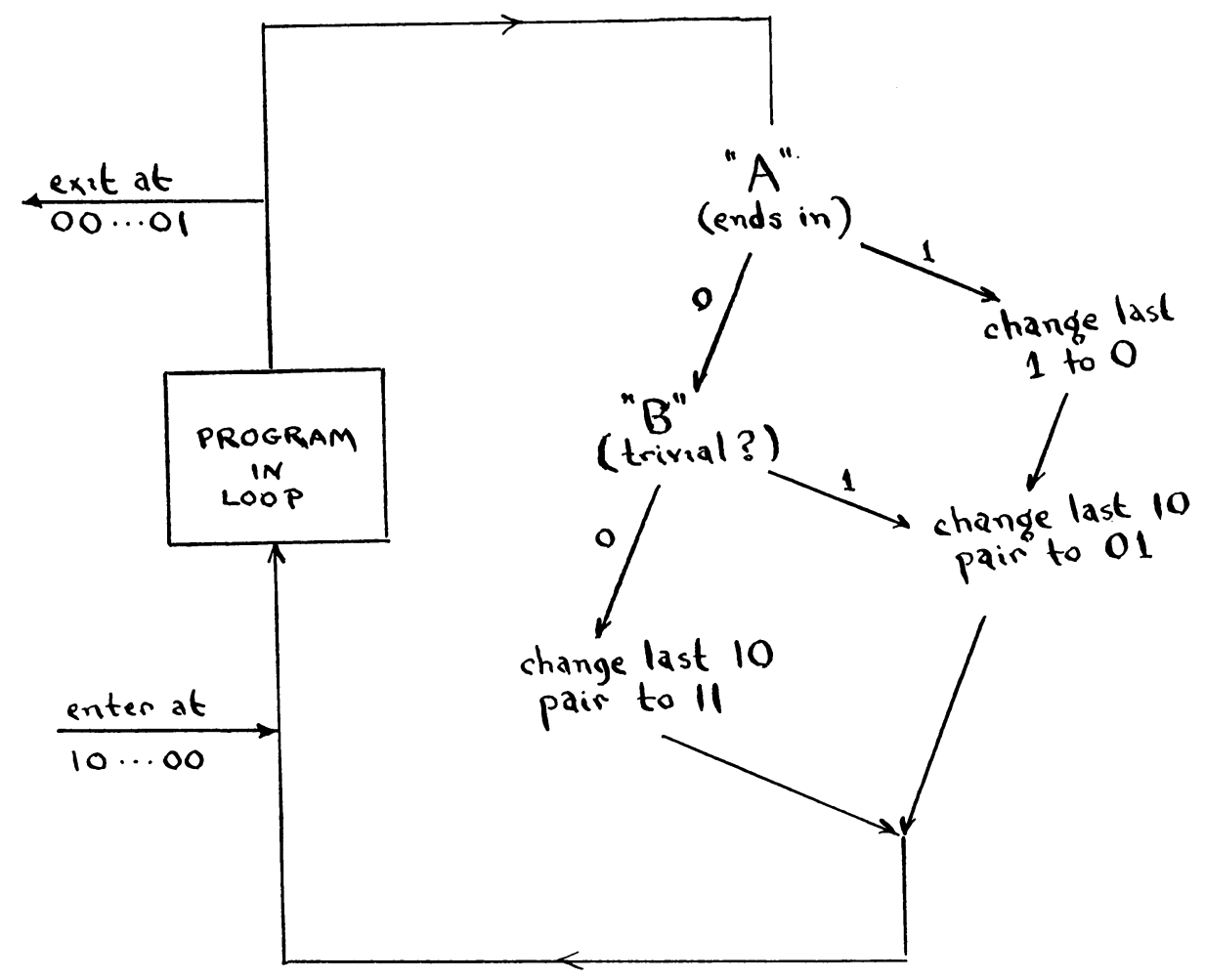

FIGURE 8

On the Boolean algebra diagram, below, we indicate the path followed if choice " $\mathrm{B}$ " is always 0 (nontrivial). From any set $C$, the path proceeds through all supersets of $C$ before going to any other set. 


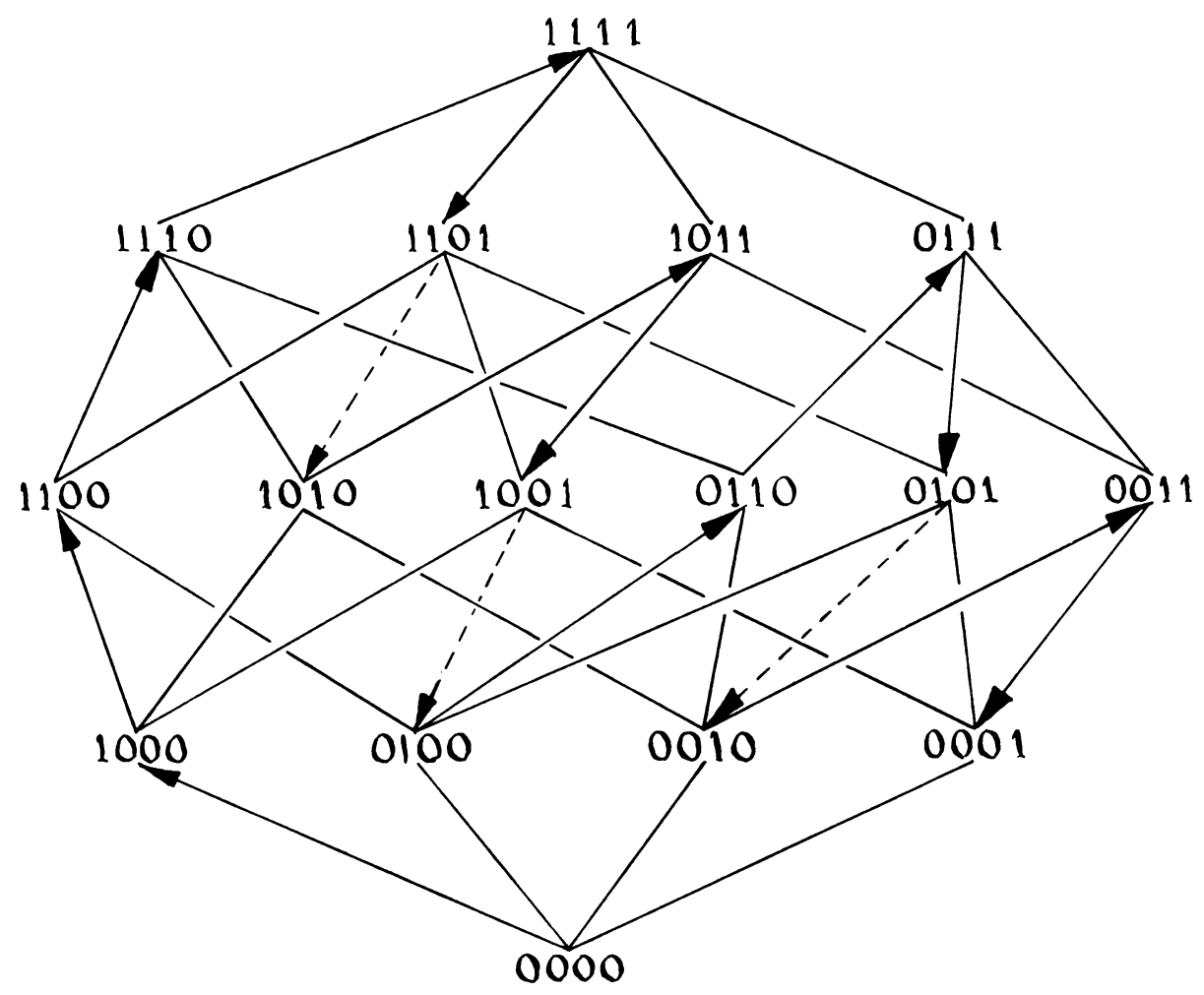

FIGURE 9

If the set 1110 produces a trivial result, the next set is 1101 (which must be checked next because it is a superset of 1100). If 0100 is trivial, the next set is 0010 . It is clear that the time saved will be substantial whenever all fairly large subsets give trivial results.

We were eventually forced to discard this procedure, in preference for the method we now describe, in which we complete partially-determined cuts to modular cuts in all possible ways. We read from left to right on each level of the lattice, and then upwards by levels. We proceed by assigning labels 0 (not in the cut) and 1 (in the cut) to each flat. Labels which are assigned by free choice are set at 1 first, and are indicated 1' ("tagged"). Having made a free choice, we work out the consequences of that choice as follows:

(a) Any flat containing a flat labeled 1 must also be labeled 1 .

(b) If a flat is labeled 0 , and is covered by a flat labeled 1 , then all other flats covering it must be labeled 0 (because the cut must be modular).

(c) If a flat is labeled 0 , then all flats contained in it must also be labeled 0 .

Having worked out the consequences of our free choice, we proceed to the next unlabeled flat, and make a free choice $1^{\prime}$.

When all flats have been labeled, a modular cut is determined. We read it out, and proceed to determine the next modular cut by resetting the last freely-chosen $1^{\prime}$ equal to 0 , erasing all labels beyond that point, and proceeding as before.

The procedure is initiated by labeling all points 0 , and making a free choice of 
$1^{\prime}$ on the first line (i.e., the first flat of rank 2). It terminates when all flats have been labeled 0 .

The other subroutines, being less wasteful of computer time, are more conventional. The lattice extensions are produced from modular cuts by the procedure outlined in [1].

To see whether two geometric lattices are isomorphic, we list the copoints of each in decreasing order of cardinality, and extend these lists to include the points in some order. We endeavor to rearrange the copoints and the points in the second list until each pairwise intersection of flats in the second list agrees in cardinality with the intersection of those flats in the same positions in the first list. This can be accomplished if and only if the point-copoint incidence relations are the same, that is, if and only if the two geometries are isomorphic.

Department of Pure Mathematics

University of Waterloo

Waterloo, Ontario, Canada

1. H. H. Crapo, "Single-element extensions of matroids," J. Res. Nat. Bur. Standards Sect. $B$, v. 69B, 1965, pp. 55-65. MR 32 \#461.

2. H. H. CRAPO \& G.-C. Rota, On the Foundations of Combinational Theory: Combinatorial Geometries, M.I.T. Press, Cambridge, Mass., 1970. (Preliminary edition.)

3. H. H. CRAPO, "The Tutte polynomial," Aequationes Math., v. 3, 1969, pp. 211-229. MR 41 \#6705.

4. J. Doyen, "Sur le nombre d'espaces linéaires non isomorphes de $n$ points," Bull. Soc. Math. Belg., v. 19, 1967, pp. 421-437. MR $40 \# 2562$.

5. D. A. HIGGS, "Matroids on 6, 7 elements," 1966. (Unpublished.)

6. A. W. INGLETON, "A note on independence functions and rank," J. London Math. Soc., v. 34, 1959, pp. 49-56. MR 21 \#655.

7. A. W. INGLETON, "Representation of matroids," Combinatorial Mathematics and Its Applications (Proc. Conf., Oxford, 1969), Academic Press, London, 1971, pp. 149-167. MR 43 \# 4700.

8. M. J. Piff \& D. J. A. Welsh, On the Number of Combinatorial Geometries, Mathematical Institute, University of Oxford, 1971. (Preprint.)

9. D. J. A. WELSH, "A bound for the number of matroids," J. Combinatorial Theory, v. 6, 1969, pp. 313-316. MR 39 \#87. 